\title{
ESTUDO PILOTO DA PREVALÊNCIA DO TRANSTORNO DE DÉFICIT DE ATENÇÃO E HIPERATIVIDADE ENTRE CRIANÇAS ESCOLARES NA CIDADE DO SALVADOR, BAHIA, BRASIL
}

\author{
Antonio Carlos Cruz Freire" ${ }^{1}$ Milena Pereira Pondé
}

\begin{abstract}
RESUMO - O presente estudo se propõe a estimar a prevalência do transtorno de déficit de atenção e hiperatividade (TDAH) em escolares através de inquérito com professores. Foi realizado um estudo piloto baseado no total de alunos do ensino fundamental, em escolas da rede pública e privada da cidade do Salvador, BA. O instrumento diagnóstico utilizado foi a Escala de TDAH versão para professores. Oito crianças $(5,3 \%)$ a p resentavam alta probabilidade de ter o subtipo predominantemente desatento, três escolares (2\%) apresentavam alta probabilidade de apresentar o subtipo predominantemente hiperativo/impulsivo e uma criança $(0,6 \%)$ apresentava alta probabilidade de apresentar o subtipo combinado. Crianças com TDAH podem ter sérios problemas de funcionamento social. O problema não se resume aos déficits trazidos pela doença, mas à associação co-mórbida com outros transtornos, que pode resultar em comprometimento social grave. 0 reconhecimento precoce e o manejo adequado do TDAH pode re di recionar o desenvolvimento educacional e psicossocial dessas crianças.
\end{abstract}

PALAVRAS-CHAVE: transtorno de déficit de atenção e hiperatividade, prevalência.

\begin{abstract}
A pilot study of prevalence of hyperactivity and attention deficit disorder in scholar children in Salvador, Bahia, Brazil

ABSTRACT - This study aims to estimate the prevalence of attention deficit hyperactivity disorder (ADHD) in scholar children through a teacher's questionnaire. A pilot study was conducted based in the total number of students under high school, in public and private schools in Salvador, Bahia, Brazil. The instrument used was Teacher's version of ADHS scale. Eight kids (5.3\%) were recognized as highly probability of attention deficit disorder, three ( $2 \%$ ) with high probability of subtype hyperactive/impulsive, and one $(0.6 \%)$ as combined subtype. Children with ADHD might have serious social problems, not only brought by the disord er, but also due to co morbidity with other disorders, leading to serious social withdrawn. Early diagnostic and treatment of ADHD might improve educational and psychosocial development of these kids.
\end{abstract}

KEY WORDS: attention deficit hyperactivity disorder, prevalence.

O transtorno de déficit de atenção e hiperatividade (TDAH) é o distúrbio neuropsiquiátrico mais comum na infância, estando, também, entre as doenças crônicas mais prevalentes em crianças escolares'. De acordo com o DSM-IV-TR, caracteriza-se por três grupos de sintomas cuja predominância vai definir o subtipo da doença: combinado, predominantemente desatento ou predominantemente hiperativo/impulsivo ${ }^{2}$. O predominantemente desatento é o subtipo mais comum, porém menos diagnosticado, uma vez que se trata de um transto rno mais tolerado socialmente ${ }^{3}$. Apesar de ser um transtorno comum em crianças, vem sendo progre s- sivamente mais identificado em adolescentes ${ }^{4} \mathrm{e}$ adultos ${ }^{5}$, significando que a doença permanece após o final da adolescência, não sendo restrita à infância. Crianças com TDAH podem ter sérios problemas de funcionamento social, incluindo dificuldades escolares, problemas de relacionamento familiar e com amigos, além de baixa auto-estima ${ }^{6,7}$. O problema do TDAH não se resume aos déficits trazidos pela doença, mas à associação co-mórbida com o u trostranstornos, que pode resultar em comprometimento social grave e exclusão. Cerca de 35\% das crianças que têm TDAH possuem algum outro transtomopsiquiátrico, sendo que a co-morbidade

\footnotetext{
${ }^{1}$ Acadêmico do Curso Médico da Escola Bahiana de Medicina e Saúde Pública (EBMSP) Salvador BA, Brasil; ${ }^{2}$ Professora Adjunta de Fa rmacologia e Psiquiatria da EBMSP. Projeto financiado com recursos da Fundação de Amparo à Pesquisa do Estado da Bahia (FAPESB).
}

Recebido 18 Agosto 2004, recebido na forma final 25 Novembro 2004. Aceito 10 Janeiro 2005. 
com transtorno desafiante opositor está dentre as mais freqüentes ${ }^{1,8,9}$.

Estima-se que 5,5 a 8,5\% das crianças em idade escolar tenham TDAH, sendo $6,9 \%$ a média entre os diversos estudos ${ }^{1}$. A média desse transtorno em estudos feitos em comunidade é $10,3 \%$, sendo a prevalência geral entre meninos de $9,2 \%$ e 3,0\% entre meninas ${ }^{10}$. Estudos que usaram o DSM-III como critério diagnóstico estimaram uma prevalência de $6,8 \%$, enquanto aqueles que usaram o DSM-III-R estimaram a prevalência de $10,3 \%$. Trabalhos mais recentes, que utilizaram os critérios diagnósticos do DSM-IV, indicam prevalências maiore ${ }^{10}$. Um estudo realizado no Brasil utilizando o DSM-IV como critério diagnóstico estimou uma prevalência de $18 \%$ de TDAH entre estudantes de primeiro grau ${ }^{11}$. Usando o mesmo critério, um estudo americano estimou a prevalência de TDAH entre crianças em escolas elementares (entre 5 e 12 anos) de 15,9\%, sendo $3,3 \%$ para o subtipo combinado, $2,3 \%$ para o subtipo hiperativo-impulsivo e $10,3 \%$ para o subtipo desatento ${ }^{12}$. Outro estudo realizado no Brasil, usando os 18 critérios do DSM-IV, estimou a prevalência de TDAH em adolescentes (entre 12 e 14 anos) como sendo $5,8 \%{ }^{13}$.

$P$ a rece não haver influência da área geográfica nas prevalências de TDAH ${ }^{12,14}$. Alguns autores sugerem que há evidências de diferenças na prevalência do TDAH em função da situação sócio-econômica'12, porém este dado ainda é controverso, posto que alguns estudos indicam que a prevalência em extratos sociais mais baixos é maior do que aquela em extratos sociais mais elevados ${ }^{15}$, enquanto outros trabalhos evidenciam exatamente o oposto ${ }^{12}$. $O$ reconhecimento precoce do TDAH e o manejo adequado dessa condição pode re di recionar o desenvolvimento educacional e psicossocial da maioria dessas crianças ${ }^{16,17}$.

O presente estudo se propõe a estimar a prevalência do TDAH em crianças escolares na cidade do Salvador através de inquérito com os professores sobre os seus alunos, identificando assim a gravidade desse problema.

\section{MÉTODO}

Foi realizado um estudo piloto baseado no total de 237057 alunos do ensino fundamental, devidamente matriculados para o ano letivo de 2003, tanto em escolas da rede pública quanto da rede privada da cidade do Salvador, Bahia. Foi admitido que a prevalência de TDAH fosse de $10 \%$, intervalo de confiança de $95 \%$ e erro assumido de $3 \%$ a fim de realizar o cálculo amostral, chegando-se ao número de 763 crianças. A partir da lista das escolas públicas da cidade do Salvador, fornecida pela Secretaria de Edu- cação do Estado da Bahia, selecionou-se aleatoriamente uma escola. Foram realizadas reuniões com professores e diretores dessa instituição para definir a participação voluntária na pesquisa e assinatura do termo de consentimento informado. Todos os alunos matriculados, da primeira à quarta série do ensino fundamental, foram incluídos neste estudo piloto, totalizando 150 crianças, o que representa $19,65 \%$ da amostra total estimada.

Para a identificação de crianças suspeitas foi utilizado como instrumento diagnóstico a Escala de Transtomo de Déficit de Atenção e Hiperatividade versão para professores ${ }^{18}$. Essa escala destina-se a avaliar os sintomas comportamentais do TDAH no contexto escolar, tendo o p rofessor como fonte de informação, levando-se em conta que o profissional necessita das informações coletadas com os pais e/ou com os professores para realizar o diagnóstico. O instrumento possui 49 itens, subdivididos em quatroFatores que avaliam o TDAH no contexto escolar: Fator 1 - Déficit de atenção (16 itens); Fator 2 - Hiperatividade / Impulsividade (12 itens); Fator 3 - Problemas de A p rendizagem (14 itens); Fator 4 - Comportamento Anti - Social (sete itens). A escala é do tipo Likert de seis pontos, organizada de modo que o professor deve assinalar: DT (discordo totalmente), D (discordo), DP (discordo parcialmente), CP (concordo parcialmente), C (concordo) e CT (concordbtotalmente). Cada resposta recebe uma pontuação que pode variar de um a seis. Após encontrar o resultado de cada item, somam-se os valores, e assim encontra-se um resultado bruto para cada Fator. Esses resultados são transformados, então, em percentil, através de tabelas de correlação, que constam no manual de instruções da escala ${ }^{18}$. Os percentis até 25 indicam que a criança apresenta menos problemas para o Fator analisado do que a maioria, sendo classificada como abaixo da expectativa. Os percentis situados entre 26 e 75 indicam que o escolar encontra-se na média, ou dentro da expectativa. Entre os percentis 76 e 94 sugere-se que a criança apresenta mais problemas que a maioria, sendo classificada como acima da expectativa. Acima do percentil 95 o escolar encontra-se na região onde há maior probabilidade de apresentar o transtorno, sendo classificado como alta probabilidade. A escolha dessa escala como instrumento de avaliação se deveu a dois fato res: trata-se de um instrumento validado no Brasil para aplicação com professores e permite evidenciar condutas que podem ser omitidas por técnicas de observação direta. Além disso, o instrumento permite não só identificar a alta probabilidade da existência de TDAH como o grupo de sintomas mais prevalentes: impulsividade, hiperatividade, déficit de atenção e comportamento anti-social. Todos os alunos foram avaliados por seus respectivos mestres. Após a devolução, as escalas que tiveram espaços deixados em branco ou que apresentavam incongruências nas respostas foram devolvidas aos respectivos professores para correção.

As informações foram armazenadas em um banco de dados, utilizando o programa estatístico epi info 2002. 
A análise das variáveis demográficas e variáveis referentes ao TDAH foram realizadas através do programa estatístico STATA Statistical Software 2001.

\section{RESULTADOS}

Foram avaliadas 150 crianças no período de maio a julho de 2003. A idade variou de 6 a 15 anos; $54,7 \%$ encontravam-se na faixa etária de 6 a 9 anos e 55,3\% entre 10 e 15 anos. Com relação ao gênero, 56 crianças $(37,3 \%)$ eram do sexo feminino e $94(62,7 \%)$ do masculino. Trinta e nove $(26 \%)$ crianças estavam cursando a primeira série, $43(28,7 \%)$ a segunda, $47(31,3 \%)$ a terceira e $21(14 \%)$ a quarta série do primeiro grau do ensino fundamental.

$O$ instrumento de avaliação prevê que, antes do p reenchimento da escala, o professor forneça a sua impressão inicial a respeito do aluno. Para os casos diagnosticados como alta-probabilidade de déficit de atenção houve um índice de discordância de $47 \%$ entre a impressão inicial do mestre e o resultado final da análise dos dados contidos na escala. Já para os casos de alta probabilidade de hiperatividade/impulsividade e de problemas de aprendizagem houve $100 \%$ de concordância.

A análise dos escores das quatro subescalas indica que 27 escolares (18\%) apresentavam mais problemas que a maioria das crianças, em relação ao subtipo predominantemente desatento, sendo classificados como acima da expectativa, enquanto que oito $(5,3 \%)$ escolares apresentavam alta probabi- lidade de apresentar o transtorno (Tabela 1). Desses seis são do sexo masculino e dois do feminino e todos estavam no intervalo de idade entre seis e nove anos. Todas crianças apresentavam problemas de a p rendizagem. Os sintomas descritos a seguir apareciam em todas elas: baixo rendimento em português; dificuldades em problemas matemáticos; raciocínio lógico lento; erros ao escrever e leitura im pe rfeita. Três crianças, apresentavam comport amento anti-social, com os sintomas (1) irrita outras crianças; (2) é briguento e (3) causa confusão em sala de aula, apareceram com maior freqüência e intensidade.

Em relação a hiperatividade/impulsividade, 22 escolares $(14,6 \%)$ apresentavam mais problemas que a maioria das crianças, sendo classificados como acima da expectativa para o transtorno. Três escolares $(2 \%)$ apresentavam alta probabilidade de apresentar o subtipo predominantemente hiperativo/impulsivo, sendo as três do sexo feminino. Uma dessas crianças tem problema de aprendizagem (Tabela 2). Os sintomas que apareceram com maior freqüência e intensidade foram: (1) baixo rendimento em português; (2) dificuldades em problemas matemáticos; (3) dificuldades de expressão e (4) raciocínio lógico lento. Os três escolares apresentavam comportamento anti-social, sendo os sintomas mais freqüentes e intensos os de causar confusão em sala de aula e ser briguento.

Tabela 1. Características das crianças classificadas como tendo alta probabilidade ou acima da expectativa para transtorno de déficit de atenção.

\begin{tabular}{lcccc}
\hline Déficit de Atenção & \multicolumn{2}{c}{ Alta Probabilidade } & \multicolumn{2}{c}{ Acima da Expectativa } \\
& N & $\%$ & N & $\%$ \\
\hline Sem hiperatividade & 8 & 88,88 & 27 & 67,5 \\
Com hiperatividade & 1 & 11,12 & 13 & 32,5 \\
Com problemas de aprendizagem & 8 & 88,88 & 31 & 77,5 \\
Com comportamento anti-social & 3 & 33,33 & 12 & 30,0 \\
Total & 9 & 100 & 40 & 100 \\
\hline
\end{tabular}

Tabela 2. Características das crianças classificadas como tendo alta probabilidade ou acima da expectativa para hiperatividade / impulsividade.

\begin{tabular}{lcccc}
\hline Hiperatividade/Impulsividade & \multicolumn{2}{c}{ Alta Probabilidade } & \multicolumn{2}{c}{ Acima da Expectativa } \\
& N & $\%$ & N & $\%$ \\
\hline Sem déficit de atenção & 3 & 75 & 22 & 62,85 \\
Com déficit de atenção & 1 & 25 & 13 & 37,15 \\
Com problemas de aprendizagem & 1 & 25 & 11 & 31,42 \\
Com comportamento anti-social & 3 & 75 & 16 & 45,71 \\
Total & 4 & 100 & 35 & 100 \\
\hline
\end{tabular}


$\mathrm{Na}$ avaliação do subtipo combinado, 13 escolares $(8,6 \%)$ apresentavam mais problemas que a maioria das crianças, sendo então classificadas como acima da expectativa para o transtorno. Apenas uma criança $(0,6 \%)$, sexo feminino, apresentava alta probabilidade de apresentar o subtipo combinado. Em associação, apresentava problemas de aprendizagem, porém sem sintomas de comportamento anti-social.

\section{DISCUSSÃO}

O TDAH é a enfermidade neuropsiquiátrica mais comum da infância, sendo mais freqüente do que paralisia cerebral, epilepsia e retardo mental. A estimativa da sua prevalência varia de acordo com o critério diagnóstico utilizado ${ }^{1,11,15,20}$. No presente estudo, estimou-se que 12 crianças (8\%) apresentavam alta probabilidade de ter TDAH, sendo que oito $(5 \%)$ apresentavam o subtipo desatento, três ( $2 \%$ ) o subtipo hiperativo/impulsivo e uma $(0,66 \%)$ o subtipo combinado, o que está de acordo com o estimado em estudos internacionais, cuja média é $6,9 \%^{1}$. Ao analisarmos as crianças que apresentavam mais problemas que a maioria dos escolares, ou seja classificadas pela escala como pertencentes ao grupo "acima da expectativa", estimou-se uma prevalência de $41 \%$, sendo $18 \%$ para o subtipo desatento, $14,7 \%$ subtipo hiperativo/impulsivo e $8,7 \%$ subtipo combinado.

Em estudos publicados, prevalências mais elevadas encontradas quando se utiliza os critérios do DSM-IV parecem se dever ao uso apenas da lista sobre sintomas atuais, desprezando outros critérios essenciais como comprometimento, início, duração e gravidade dos sintomas, bem como a exclusão de outros transtornos. A prevalência de $16 \%$ estimada sem o uso do critério comprometimento reduziu-se para $6,8 \%$ quando esse critério foi utilizado ${ }^{10}$. Num estudo feito na Austrália, estimou-se a prevalência de $14,7 \%$ de TDAH com o uso apenas da lista de sintomas do DSM-IV. No mesmo estudo, ao se utilizar também os outros critérios, a prevalência de TDAH em crianças de 6 a 12 anos foi 9,4\% ${ }^{19}$.

Apesar do instrumento utilizado no presente estudo, avaliar os sintomas comportamentais do TDAH no contexto escolar, não foram identificadas prevalências elevadas. Isto pode se dever ao fato de que, para um escolar ser classificado como tendo alta probabilidade de apresentar o transtorno, é preciso que obtenha pontuação máxima ou muito próxima da máxima possível para o Fator avaliado, indicando uma alta especificidade da escala.
O diagnóstico do TDAH em crianças se baseia em uma história obtida com pais, professores ou outros cuidadores, não requerendo que o médico testemunhe os sintomas ou os obtenha diretamente das crianças. A avaliação diagnóstica mais utilizada é o uso de escalas de classificação de pais e professores. Num estudo realizado no Canadá, a taxa de prevalência de TDAH estimada por instrumentos utiliza dos por professores foi $13,2 \%$, sendo de $7,3 \%$ quando estimada por instrumentos utilizados pelos pais ${ }^{14}$.

A prevalência do subtipo desatento neste estudo está de acordo com os dados da literatura, uma vez que a maior proporção de crianças identificadas como tendo alta probabilidade de terem o TDAH pertenceram a este grupo ${ }^{12,17}$. Apenas uma criança, com alta probabilidade de ter o transtorno, pertenceu ao subtipo clínico combinado, enquanto três escolares pertenceram ao subtipo clínico predominantemente hiperativo, sendo esta apresentação diversa da que é descrita em outros estudos, nos quais o subtipo combinado é mais prevalente do que o subtipo predominantemente hiperativo ${ }^{12,15}$. O mesmo perfil de distribuição dos subtipos clínicos é observado para os escolares classificados no grupo acima da expectativa.

A predominância do desenvolvimento do TDAH nos indivíduos do sexo masculino não foi obsenvada neste estudo, discordando da literatura ${ }^{15,20}$. Na população geral, 9,2\% (5,8\%-13,6\%) das crianças do sexo masculino e $2,9 \%$ (1,9\%-4,5\%) das crianças do sexo feminino apresentam achados comportamentais consistentes com o diagnóstico de TDAH ${ }^{1}$. No grupo de escolares classificados como acima da expectativa, houve predominância do gênero masculino em relação ao feminino, em proporção de 1,8: 1. Ao analisar os subtipos de acordo com o sexo, obsena-se maior prevalência do subtipo desatento entreos meninos e de hiperatividade entre as meninas, no grupo com alta probabilidade de apresentar o TDAH, diferindo de dados da literatura, na qual o oposto foi descrito ${ }^{15}$. No entanto, ao anali$s$ a rmos as crianças classificadas no grupo acima da expectativa, observamos maior prevalência do subtipo desatento entre as meninas e de hiperatividade entre os meninos. Desta forma, pode ser que a maior prevalência encontrada em meninas se refira especificamente aos casos mais graves, uma vez que a prevalência entre as crianças pertencentes ao grupo acima da expectativa coincide com a literatura, sendo também maior entre meninos.

$P$ a rece não haver motivos para se pensar numa diferença de prevalência de TDAH em função da dis- 
tribuição geográfica, uma vez que nenhum dos estudos revisados na literatura especializada indica tal diferença. Alguns autores sugerem que há evidências de diferenças de prevalência de TDAH em função do status sócio-econômico ${ }^{12,15}$, Como existe contradição em relação à influência do nível sócioeconômico na prevalência do TDAH, a segunda fase desta pesquisa está sendo conduzida em escolas públicas e privadas, visando ajudar a elucidação das contradições existentes.

A análise dos sintomas evidencia que a corre lação entre déficit de atenção e problemas de apre ndizagem foi positiva em $88,88 \%$ dos casos, enquanto a correlação entre hiperatividade e comportamento anti-social foi positiva em $75 \%$ dos casos. Brown et al. ${ }^{10}$, investigando comorbidade com TDAH, estimaram que $35,2 \%$ das crianças com esse transto rnotêm também transtorno desafiante opositor e $25,7 \%$ se associam à desordem de conduta. A prevalência de transtorno desafiante opositor estimada na população geral é 0,7 a 5,5\% e a de desordem de conduta é 0,2 a 2,3\%. ${ }^{14}$ Entreescolares a prevalência estimada de transtorno desafiante opositor é $4,5 \%$ e a de desordem de conduta é $2,5 \%{ }^{12}$. Esses resultados indicam uma prevalência muito maior dos dois tipos de distúrbio de comportamento disruptivo entre crianças com TDAH do que em crianças da população geral. O subtipo desatento tem muito menos distúrbio de comportamento disru ptivo do que os hiperativos/impulsivos ou os subtipos combinados $^{10}$, o que está de acordo com os achados deste estudo, cuja correlação entre o subtipo predominantemente desatento e comportamento antisocial foi $37,5 \%$.

Dos nove $(6 \%)$ escolares que apresentam alta probabilidade de apresentar déficit de atenção, cinco foram identificados por seus respectivos professoresem uma avaliação inicial como tendo dificuldades de atenção ou concentração, enquanto 4 crianças não foram reconhecidas pelos docentes como desatentos. Em relação às crianças hiperativas, na avaliação inicial, todas as que foram identificadas pelos docentes como agitadas estavam também classificadas através do instrumento como tendo alta probabilidade de ter o transtorno. Esses dados sugerem que, para as crianças nas quais prepondera o déficit de atenção, o diagnóstico é dificultado por se tratar de um transtorno mais tolerado socialmente, o que pode retardar o acesso ao tratamento.

\section{REFERÊNCIAS}

1. American Academy of Pediatrics (AAP). Clinical practice guideline: diagnosis and evaluation of the child with attention-deficit/hyperactivity disorder. Pediatrics 2000;105:1158-1170.

2. American Psychiatric Association. Diagnostical and statistical manual of mental Disorders; 4.Ed. Washington, DC: American Psychiatric Association, 2000

3. Fisher BC. Attention defict disorder misdiagnosis New York: CRC Press, 1998.

4. Biederman J, Faraone SV, Milberger S, et al. Predictors of persistence and remissions of ADHD into adolescence: results from a four-year prospective follow-up study. J. Am Acad Child Adolesc Psychiatry 1996;3 5:343-351.

5. Biederman J, Faraone SV, Spencer T, et al. Patterns of psychiatric comorbidity, cognition, and psychosocial functioning in adults with attention deficit hyperactivity disorder. Am J Psychiatry 1993;150:1792-1798.

6. Schachar R, Taylor E, Wieselberg MB, Ghorley G, Rutter M. Changes in family functioning and relationships in children who respond to methylphenidate J Am Acad Child Adolesc Psychiatry 1987;26:728-732.

7. Almond BW Jr, Tanner JL, Goffman HF. The family is the patient: using family interviews in children's medical care, 2.Ed. Baltimore: Williams \& Wilkins, 1999:307-313.

8. Kadesjü B, Gillberg C. The comorbity of ADHD in the general population of Swedish school-age children. J Child Psychol Psychiatry 2001;42:487-492.

9. Souza I, Serra MA, Mattos P, Franco VA. Comorbidade em crianças e adolescentes com transtorno de déficit de atenção. A rqNeuropsiquiatr 2001;59:401-406.

10. Brown R, Freeman WS, Perrin JM, et al. Prevalence and assessment of attention-deficit/hyperactivity disorder in primary care settings. Pediatrics 2001;107:E43.

11. Guardiola A, Fuchs FD, Rotta NT. Prevalence of attention-deficit hyperactivity disorders in students. Arq Neuropsiquiatr 2000;58:401-407.

12. Nolan EE, Gadow KD, Sprafkin J. Teacher Reports of DSM-IV ADHD, ODD, and CD symptoms in schoolchildren. J Am Acad Child Adolesc Psychiatry 2001;40:241-249.

13. Rohde LA, Biederman J, Busnello EA, et al. ADHD in a school sample of Brazilian adolescents: a study of prevalence, comorbid conditions, and impairments. J Am Acad Child Adolesc Psychiatry 1999;38:716-722.

14. Breton JJ, Bergeron L, Valla JP, Bertiaume C, Gaudet N. Quebec child mental health survey: prevalence of DSM-III-R mental health disorders. J Child Psychol Psychiatry 1999;40:375-84.

15. Pineda DA, Lopera F, Henao GC, Placio JD, Castellanos FX. Confirmación de la alta prevalencia del transtorno por déficit em uma cominidad colombiana. Rev Neurol 2001;32:217-222.

16. Baumgaertel A, Copeland L, Wolraich ML. Attention deficit-hyperactivity disorder. In Disorders of development and learning: a practical guide to assessment and Management, 2.Ed. St Louis: Mosby Yearbook, 1996:424-456.

17. Cantwell DP. Attention deficit disorder: a review of the past 10 years. J Am Acad Child Adolesc Psychiatry 1996;35:978-987.

18. Benczic EBP. Manual da escala de transtorno de déficit de atenção e hiperatividade. São Paulo: Casa do Psicólogo, 2000.

19. Graetz BW, Sawyer MG, Hazell FL, Arney F, Baghurst P. Validity of DSMIV ADHD subtypes in a nationally representative sample of Australian children and adolescents. J Am Acad Child Adolesc Psychiatry 2001; 40:1410-1417.

20. Montiel-Nava C, Peña JA, Motiel-Barbero I. Datos epidemiológicos del transtorno por déficit de atención con hiperactividad en una muestra de niños Marabinos. Rev Neurol 2003;37:815-819.

21. Zentall SS. Research on the educational implications of attention deficit hyperactivity disorder. Exceptional Child 1993;60:143-153.

22. Ward MF, Wender PH, Reimherr FW. The Wender Utah Rating Scale: an aid in the retrospective diagnosis of childhood attention deficit hyperactivity disorder, Am J Psychiatry.1993;150:1280. 